\title{
A Numeration System for Fibonacci-like Wang Shifts
}

\author{
Sébastien Labbé ${ }^{1}$ and Jana Lepšová ${ }^{1,2}$ \\ 1 Univ. Bordeaux, CNRS, Bordeaux INP, LaBRI, UMR 5800, F-33400, Talence, France \\ 2 FNSPE, CTU in Prague, Trojanova 13, 12000 Praha, Czech Republic \\ sebastien.labbe@labri.fr, jana.lepsova@labri.fr
}

\begin{abstract}
Motivated by the study of Fibonacci-like Wang shifts, we define a numeration system for $\mathbb{Z}$ and $\mathbb{Z}^{2}$ based on the binary alphabet $\{0,1\}$. We introduce a set of 16 Wang tiles that admits a valid tiling of the plane described by a deterministic finite automaton taking as input the representation of a position $(m, n) \in \mathbb{Z}^{2}$ and outputting a Wang tile.
\end{abstract}

\section{Introduction}

A theorem of Cobham (1972) says that a sequence $u=\left(u_{n}\right)_{n \geq 0}$ is $k$-automatic with $k \geq 2$ if and only if it is the image, under a coding, of a fixed point of a $k$-uniform morphism [AS03, Theorem 6.3.2]. This result was extended to non-uniform morphisms [RM02], see also [BR10, Theorem 3.4.1], by replacing the usual base- $k$ expansion of nonnegative integers by an abstract numeration system and a regular language [LR01]. It was later extended to configurations $x: \mathbb{N}^{d} \rightarrow \Sigma$ in dimension $d \geq 1$ based on the notion of shape-symmetric morphic words [CKR10], see also [BR10, Theorem 3.4.26] and [AA20, § 5].

In this article, we explore an extension of Cobham's result beyond the nonnegative octant $\mathbb{N}^{d}$ to include configurations $x: \mathbb{Z}^{d} \rightarrow \Sigma$ defined on the whole lattice $\mathbb{Z}^{d}$. We concentrate on one example in dimension $d=2$. The example is motivated by the study of Wang tilings of the plane. Given an alphabet $\mathcal{C}$ of colors, a Wang tile is a 4 -tuple $(a, b, c, d) \in \mathcal{C}^{4}$ that represents the labeling of the edges of a unit square, by convention, in the order corresponding to a positive rotation on the complex plane, i.e., $a$ is the east edge label, $b$ is the north edge label, $c$ is the west edge label and $d$ is the south edge label.

\begin{tabular}{|c|c|c|c|c|c|c|c|}
\hline $\begin{array}{l}\mathrm{J} \\
\mathrm{O}\end{array}$ & $\mathrm{H} \underset{\mathrm{L}}{\mathrm{O}} \mathrm{D}$ & $\stackrel{\mathrm{M}}{\mathrm{P}} \mathrm{J}$ & $\begin{array}{c}M \\
D \\
\mathrm{~K}\end{array}$ & $\mathrm{~J} \underset{\mathrm{P}}{\mathrm{P}} \mathrm{H}$ & $\mathrm{H}_{\mathrm{N}}^{\mathrm{P}} \mathrm{H}$ & $\mathrm{D} \underset{\mathrm{P}}{\mathrm{n}} \mathrm{H}$ & $\mathrm{I} \underset{\mathrm{O}}{7} \mathrm{~B}$ \\
\hline $\begin{array}{l}\mathrm{L} \\
8 \mathrm{I} \\
\mathrm{O}\end{array}$ & I & $\begin{array}{c}\mathrm{I} 10 \mathrm{~A} \\
\mathrm{O}\end{array}$ & $\mathrm{I}_{\mathrm{P}}^{\mathrm{I} 11 \mathrm{E}}$ & I 12 I & $\begin{array}{c}\mathrm{K} \\
\mathrm{B} 13 \mathrm{I} \\
\mathrm{M}\end{array}$ & $\mathrm{A}_{\mathrm{K}} \mathrm{I}$ & $\mathrm{I} \underset{\mathrm{P}}{\mathrm{N}}$ \\
\hline
\end{tabular}

Fig. 1. The set $\mathcal{Z}=\left\{z_{0}, \ldots, z_{15}\right\}$ of 16 Wang tiles. The index $i$ of the tile $z_{i}$ is written in the center of each tile. 
We introduce a set $\mathcal{Z}=\left\{z_{0}, \ldots, z_{15}\right\}$ of 16 Wang tiles shown in Figure 1 . The set $\mathcal{Z}$ is a simplification of an existing aperiodic set of 19 Wang tiles [Lab19] after identification of few colors, which was shown to be related [Lab21] to the smallest set of aperiodic Wang tiles found by Jeandel and Rao [JR21].

A valid configuration over the set of Wang tiles $\mathcal{Z}$ is a function $f: \mathbb{Z}^{2} \rightarrow$ $\{0, \ldots, 15\}$ such that adjacent tiles have the same label on their common edge, i.e., for every $n \in \mathbb{Z}^{2}$, the east label of the tile $z_{f(n)}$ is equal to the west label of the tile $z_{f\left(n+e_{1}\right)}$ and the north label of the tile $z_{f(n)}$ is equal to the south label of the tile $z_{f\left(n+e_{2}\right)}$. A partial valid configuration is shown in Figure 2. It is this particular configuration that is linked with a numeration system in Theorem 1. The set $\Omega_{\mathcal{Z}}$ of valid configurations $f: \mathbb{Z}^{2} \rightarrow\{0, \ldots, 15\}$ is called the Wang shift associated to the set of Wang tiles $\mathcal{Z}$.

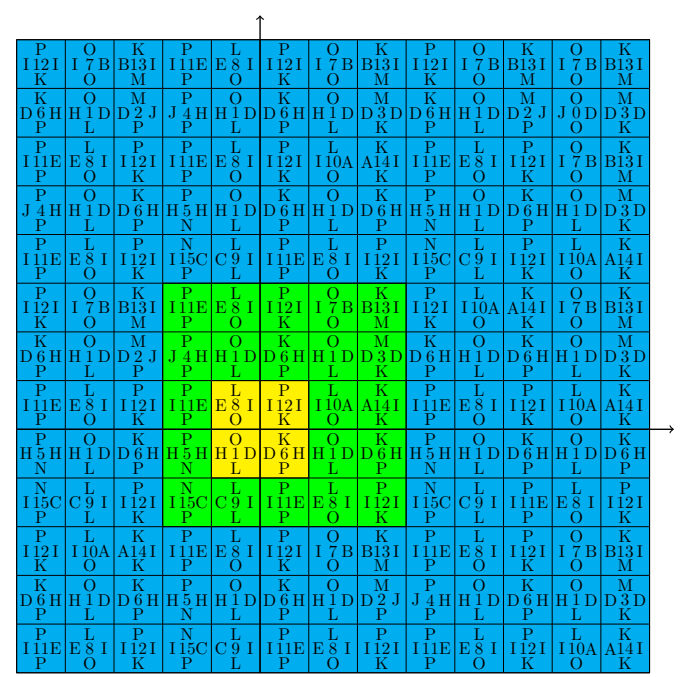

Fig. 2. A partial valid configuration $\left[-5,8\left[^{2} \rightarrow\{0, \ldots, 15\}\right.\right.$ with the set $\mathcal{Z}$ of Wang tiles.

We show the following result which states a link between a specific configuration over the set $\mathcal{Z}$ of Wang tiles shown in Figure 1, a numeration system for $\mathbb{Z}^{2}$ and a deterministic finite automaton with output (DFAO). Definition of DFAO is recalled in Section 3 and corresponds to the classic definition [AS03].

Theorem 1. Let $\mathcal{Z}$ be the set of 16 Wang tiles shown in Figure 1. There exist a valid Wang configuration $x \in \Omega_{\mathcal{Z}}$ and a DFAO $\mathcal{A}$ and a numeration system $\mathcal{F}$ for $\mathbb{Z}^{2}$ with a representation function $\operatorname{rep}_{\mathcal{F}}: \mathbb{Z}^{2} \rightarrow\left\{\left(\begin{array}{l}0 \\ 0\end{array}\right),\left(\begin{array}{l}0 \\ 1\end{array}\right),\left(\begin{array}{l}1 \\ 0\end{array}\right),\left(\begin{array}{l}1 \\ 1\end{array}\right)\right\}^{*}$ such that the tile at position $\boldsymbol{n} \in \mathbb{Z}^{2}$ in $x$ is $x_{\boldsymbol{n}}=\mathcal{A}\left(\operatorname{rep}_{\mathcal{F}}(\boldsymbol{n})\right)$.

In fact, the Wang shift $\Omega_{\mathcal{Z}}$ is self-similar, minimal and aperiodic. Moreover, it is topologically conjugate to the Wang shift $\Omega_{\mathcal{U}}$ generated by the set $\mathcal{U}$ of 19 
Wang tiles introduced in [Lab19]. These results are not shown here due to lack of space and will be part of an extended version of this article.

The article is structured as follows. In Section 2, we introduce a Fibonacci numeration system for $\mathbb{Z}$ and $\mathbb{Z}^{2}$. In Section 3, we illustrate how to change the usual automaton in Cobham's theorem for $\mathbb{N}$ so that it can read the representation of integers including the negative ones. In Section 4, we recall the definitions and notations for two-dimensional words, languages and morphisms. The selfsimilarity of the Wang shift $\Omega_{\mathcal{Z}}$ is stated in Section 5 (the proof is available in the appendix of the preprint version) from which the automaton of Theorem 1 is deduced, see Figure 5. The proof of Theorem 1 is done in Section 6 .

Acknowledgements. This work was supported by the Agence Nationale de la Recherche through the project Codys (ANR-18-CE40-0007).

\section{A Fibonacci Numeration System for $\mathbb{Z}$ and $\mathbb{Z}^{2}$}

Let $\left(F_{n}\right)_{n \geq 1}$ be the Fibonacci sequence defined with the reccurent relation

$$
F_{0}=1, F_{1}=1, F_{2}=2, F_{n+2}=F_{n+1}+F_{n} \quad \text { for all } n \geq 1 .
$$

By the Zeckendorf theorem [Zec72] every nonnegative integer $n$ can be represented as a unique sum of nonconsecutive Fibonacci numbers $n=\sum_{i=1}^{\ell} w_{i} F_{i}$, where $\ell=$ $\max \left\{i \in \mathbb{N}_{0}: F_{i} \leq n\right\}, w_{i} \in\{0,1\}$ and $w_{i} w_{i+1}=0$, for all $i \in\{1,2, \ldots, \ell-1\}$.

Inspired by the Two's complement, "the most common method of representing signed integers on computers" ${ }^{3}$ we introduce a numeration system $\mathcal{F}$ which extends the Fibonacci numeration system to all $n \in \mathbb{Z}$ as follows. For each binary word $w=w_{2 k+1} w_{2 k} \cdots w_{1} \in \Sigma^{2 k+1}$ of odd length over the alphabet $\Sigma=\{0,1\}$, we define

$$
\operatorname{val}_{\mathcal{F}}(w)=\sum_{i=1}^{2 k} w_{i} F_{i}-w_{2 k+1} F_{2 k}
$$

The following lemma is an exercise based on the Fibonacci recurrence.

Lemma 2. Let $k \in \mathbb{N}$ and $w \in \Sigma^{2 k} \backslash \Sigma^{*} 11 \Sigma^{*}$. We have

1. $\operatorname{val}_{\mathcal{F}}(0 w)=\operatorname{val}_{\mathcal{F}}(000 w)=\operatorname{val}_{\mathcal{F}}(110 w)$,

2. $\operatorname{val}_{\mathcal{F}}(1 w)=\operatorname{val}_{\mathcal{F}}(101 w)$,

3. $\operatorname{val}_{\mathcal{F}}(100 w)=\operatorname{val}_{\mathcal{F}}(000 w)-F_{2 k+2}$,

4. $0 \leq \operatorname{val}_{\mathcal{F}}(0 w)<F_{2 k+1}$,

5. $-\bar{F}_{2 k+2} \leq \operatorname{val}_{\mathcal{F}}(100 w)<0$.

Thus, the first digit of $w \in \Sigma^{2 k+1} \backslash \Sigma^{*} 11 \Sigma^{*}$ gives the sign (nonnegative or negative) of the value $\operatorname{val}_{\mathcal{F}}(w)$. We can show the following.

Proposition 3. For every $n \in \mathbb{Z}$ there exists a unique odd-length word

$$
w \in \Sigma(\Sigma \Sigma)^{*} \backslash\left(\Sigma^{*} 11 \Sigma^{*} \cup 000 \Sigma^{*} \cup 101 \Sigma^{*}\right)
$$

such that $n=\operatorname{val}_{\mathcal{F}}(w)$.

\footnotetext{
$\overline{3}$ https://en.wikipedia.org/wiki/Two's_complement
} 
Proof. (Unicity). Let $w, w^{\prime} \in \Sigma(\Sigma \Sigma)^{*} \backslash\left(\Sigma^{*} 11 \Sigma^{*} \cup 000 \Sigma^{*} \cup 101 \Sigma^{*}\right)$ of minimal length such that $\operatorname{val}_{\mathcal{F}}(w)=\operatorname{val}_{\mathcal{F}}\left(w^{\prime}\right)$. If $w \in 1 \Sigma^{*}$, then $\operatorname{val}_{\mathcal{F}}(w)=\operatorname{val}_{\mathcal{F}}\left(w^{\prime}\right)<0$ and $w^{\prime} \in 1 \Sigma^{*}$ as well. In fact, we must have $w, w^{\prime} \in 100 \Sigma^{*}$. Thus $w=10 u$ and $w=10 u^{\prime}$ for some words $u, u^{\prime} \operatorname{such}$ that $\operatorname{val}_{\mathcal{F}}(u)=\operatorname{val}_{\mathcal{F}}\left(u^{\prime}\right)$. This contradicts the minimality of the lengths of $w$ and $w^{\prime}$. If $w \in 0 \Sigma^{*}, \operatorname{then} \operatorname{val}_{\mathcal{F}}(w)=\operatorname{val}_{\mathcal{F}}\left(w^{\prime}\right) \geq 0$ and $w^{\prime} \in 0 \Sigma^{*}$ as well. But $w, w^{\prime} \notin 000 \Sigma^{*}$, thus $w \in\{01,001\} u$ and $w^{\prime} \in$ $\{01,001\} u^{\prime}$ for some $u, u^{\prime} \in \Sigma^{*}$. From Zeckendorf's theorem applied to $1 u$ and $1 u^{\prime}$, we conclude that $u=u^{\prime}$.

(Existence). If $n=0$, then $n=0=\operatorname{rep}_{\mathcal{F}}(0)$. Assume that $n>0$. From Zeckendorf's theorem, there exists a unique $u \in 1 \Sigma^{*} \backslash \Sigma^{*} 11 \Sigma^{*}$ such that $n=$ $\operatorname{val}_{F}(u)$. If $u$ has odd-length, then $n=\operatorname{val}_{\mathcal{F}}(00 u)$. If $u$ has even-length, then $n=$ $\operatorname{val}_{\mathcal{F}}(0 u)$. Now assume that $n<0$. Let $k \in \mathbb{N}$ be such that $-F_{2 k} \leq n<-F_{2 k-2}$. We have $0 \leq n+F_{2 k}<F_{2 k}-F_{2 k-2}=F_{2 k-1}$. Let $w \in \Sigma^{2 k-2} \backslash \Sigma^{*} 11 \Sigma^{*}$ such that $\operatorname{val}_{\mathcal{F}}(000 w)=n+F_{2 k}$. We thus have $n=\operatorname{val}_{\mathcal{F}}(100 w)$.

Definition 4 (Numeration system $\mathcal{F}$ for $\mathbb{Z}$ ). For each $n \in \mathbb{Z}$, we denote by $\operatorname{rep}_{\mathcal{F}}(n)$ the unique word satisfying the proposition.

The numeration system $\mathcal{F}$ is illustrated in Figure 3.

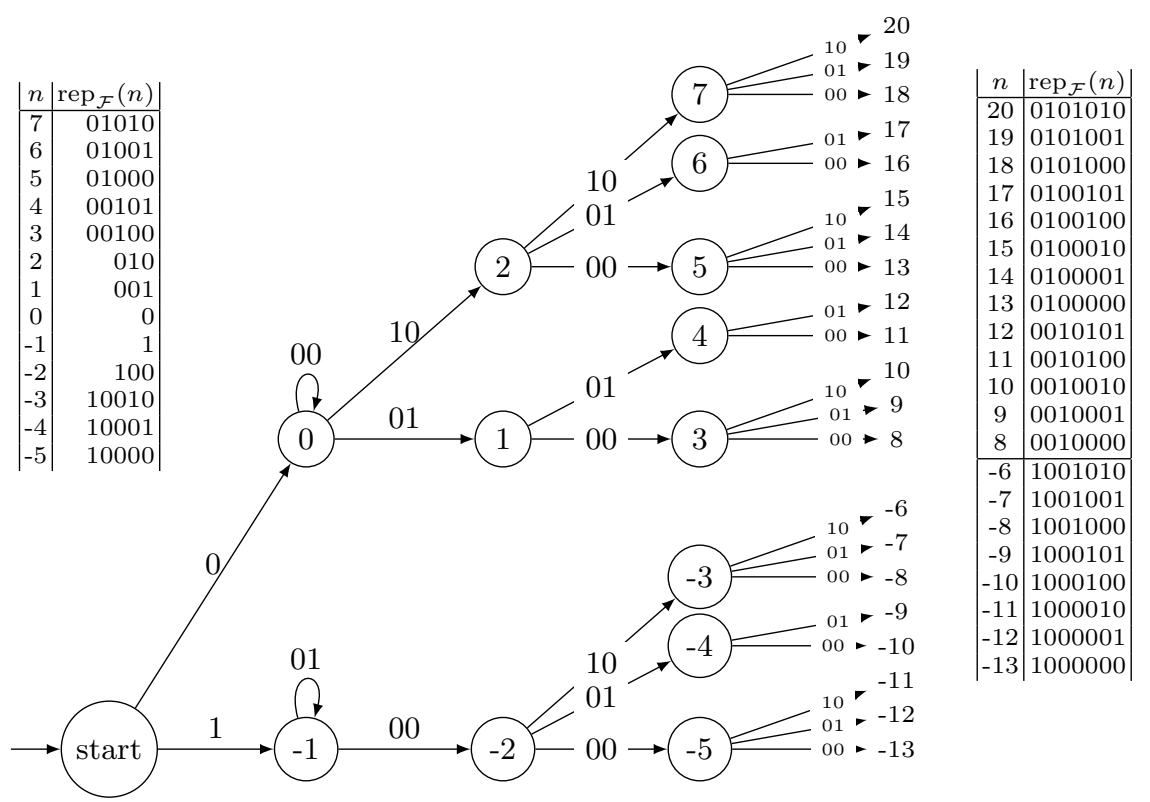

Fig. 3. Representations in the numeration system $\mathcal{F}$ of $n \in[-13,21[$.

We now extend that numeration system to $\mathbb{Z}^{2}$. If $\boldsymbol{n}=\left(n_{1}, n_{2}\right) \in \mathbb{Z}^{2}$ is such that $\left|n_{1}\right|>>\left|n_{2}\right|$, then the word representing $n_{2}$ is smaller than the word 
representing $n_{1}$. We handle this issue by padding the smaller word so that it becomes of the same length as the longer one. The padding is done differently according to the sign of the number involved: nonnegative numbers are padded with 00 , while negative numbers are padded with 10 . This is consistent with the numeration system $\mathcal{F}$, refer also to the two loops in Figure 3.

Definition 5 (Numeration system $\mathcal{F}$ for $\mathbb{Z}^{2}$ ). Let $\boldsymbol{n}=\left(n_{1}, n_{2}\right) \in \mathbb{Z}^{2}$. We define

$$
\operatorname{rep}_{\mathcal{F}}(\boldsymbol{n})=\left(\begin{array}{c}
\operatorname{pad}_{t}\left(\operatorname{rep}_{\mathcal{F}}\left(n_{1}\right)\right) \\
\operatorname{pad}_{t}\left(\operatorname{rep}_{\mathcal{F}}\left(n_{2}\right)\right)
\end{array}\right)
$$

where $t=\max \left\{\left|\operatorname{rep}_{\mathcal{F}}\left(n_{1}\right)\right|,\left|\operatorname{rep}_{\mathcal{F}}\left(n_{2}\right)\right|\right\}$ and

$$
\operatorname{pad}_{t}(w)= \begin{cases}(00)^{\frac{1}{2}(t-|w|)} w & \text { if } w \in 0 \Sigma^{*}, \\ (10)^{\frac{1}{2}(t-|w|)} w & \text { if } w \in 1 \Sigma^{*} .\end{cases}
$$

The set of all canonical representations $\operatorname{rep}_{\mathcal{F}}(\boldsymbol{n})$ for $\boldsymbol{n} \in \mathbb{Z}^{2}$ are words in $\left\{\left(\begin{array}{l}0 \\ 0\end{array}\right),\left(\begin{array}{l}0 \\ 1\end{array}\right),\left(\begin{array}{l}1 \\ 0\end{array}\right),\left(\begin{array}{l}1 \\ 1\end{array}\right)\right\}^{*}$ of odd length such that there are no consecutive one's in each row. E.g. $\operatorname{rep}_{\mathcal{F}}(-2,9)=\left(\begin{array}{l}1010100 \\ 0010001\end{array}\right), \operatorname{rep}_{\mathcal{F}}(14,2)=\left(\begin{array}{c}0100001 \\ 0000010\end{array}\right)$. The length of the representation splits $\mathbb{Z}$ and $\mathbb{Z}^{2}$ into levels.

Lemma 6. For every $k \in \mathbb{N}$, we have

$$
\begin{aligned}
& \left\{n \in \mathbb{Z}:\left|\operatorname{rep}_{\mathcal{F}}(n)\right|=2 k+1\right\}=I_{k} \backslash I_{k-1}, \\
& \left\{\boldsymbol{n} \in \mathbb{Z}^{2}:\left|\operatorname{rep}_{\mathcal{F}}(\boldsymbol{n})\right|=2 k+1\right\}=I_{k}^{2} \backslash I_{k-1}^{2}
\end{aligned}
$$

where $I_{k}=\left\{i \in \mathbb{Z} \mid-F_{2 k} \leq i<F_{2 k+1}\right\}$ for $k \geq 0$ and $I_{-1}=\varnothing$.

Proof. The first equality follows from the fact that $I_{k}=\left\{n \in \mathbb{Z}:\left|\operatorname{rep}_{\mathcal{F}}(n)\right| \leq\right.$ $2 k+1\}$ where the minimal value $-F_{2 k}$ is attained by the word $1(00)^{k}$ and the maximal value $F_{2 k+1}-1$ is attained by the word $0(10)^{k}$. The second equality follows from the fact that $I_{k}^{2}=\left\{\boldsymbol{n} \in \mathbb{Z}^{2}:\left|\operatorname{rep}_{\mathcal{F}}(\boldsymbol{n})\right| \leq 2 k+1\right\}$.

In Figure 2, the levels $I_{0}^{2} \backslash I_{-1}^{2}, I_{1}^{2} \backslash I_{0}^{2}$ and $I_{2}^{2} \backslash I_{1}^{2}$ are shown in yellow, green and blue respectively.

\section{An Automaton not only for Nonnegative Integers}

We introduce the terms based on [BR10] to be used in this section. Let $\sigma$ : $A \mapsto A^{*}$ be a non-erasing morphism prolongable on the letter $a \in A$ such that $x=\left(x_{n}\right)_{n \geq 0}=\sigma^{\omega}(a)$ is infinite. Let $C=\left\{0, \ldots, \max _{b \in A}|\sigma(b)|-1\right\}$ be an alphabet. The deterministic finite automaton with output (DFAO) associated to the morphism $\sigma$ and letter $a$ is the 5 -tuple ${ }^{4} \mathcal{A}_{\sigma, a}=(A, C, \delta, a, A)$, where $\delta: A \times C \rightarrow A$ is a partial function such that $\delta(b, i)=c$ if and only if $c=u_{i}$ and $\sigma(b)=u_{0} \ldots u_{|\sigma(b)|-1}$. Let $L$ be the language accepted by $\mathcal{A}_{\sigma, a}$. Then the

\footnotetext{
${ }^{4}$ In contrast to [BR10] we omit the coding as it is the identity map.
} 
triple $\mathcal{S}=\left(L \backslash 0 C^{*}, C,<\right)$ is an abstract numeration system, where $(C,<)$ is the totally ordered alphabet with the natural order on $\mathbb{N}$ and the language $L \backslash 0 C^{*}$ is radix ordered. Radix order $<_{\text {rad }}$ is defined for words $u, v \in L \backslash 0 C^{*}$ as follows: $u<_{\text {rad }} v$ if and only if $|u|<|v|$ or $|u|=|v|$ and $u<_{\text {lex }} v$. The map $\operatorname{rep}_{\mathcal{S}}: \mathbb{N} \rightarrow L \backslash 0 C^{*}$ maps $n \in \mathbb{N}$ to the $(n+1)^{t h}$ word in the language $L \backslash 0 C^{*}$ and the map $\operatorname{val}_{\mathcal{S}}: L \rightarrow \mathbb{N}$ maps a word $w$ to the number $n$ such that $\operatorname{rep}_{\mathcal{S}}(n)=w^{\prime}$, where $w=0^{p} w^{\prime}$ for a $p \geq 0$. For some state $r \in A$ and word $w \in C^{*}$, we denote by $\mathcal{A}_{\sigma, a}(r, w)$ the state reached by the automaton after following the path labeled by $w$ from the state $r$. We denote it by $\mathcal{A}_{\sigma, a}(w)$ when $r$ is the initial state. The following is essentially a reformulation of [BR10, Corollary 3.4.14].

Proposition 7. For every integer $n>0$, there exist integers $m \in \mathbb{N}$ and $\ell \in C$ such that $x_{n}=\sigma\left(x_{m}\right)[\ell]$ and $\operatorname{rep}_{\mathcal{S}}(n)=\operatorname{rep}_{\mathcal{S}}(m) \cdot \ell$. Moreover, for any $i \in \mathbb{N}$, $\left|\operatorname{rep}_{\mathcal{S}}(n)\right|=i$ if and only if $\left|\sigma^{i-1}(a)\right| \leq n<\left|\sigma^{i}(a)\right|$.

Proof. Let $n \in \mathbb{N}$. Let $u \in L \backslash 0 C^{*}$ such that $\operatorname{rep}_{\mathcal{S}}(n)=u$. Let $w \in C^{*}$ and $\ell \in C$ such that $u=w \ell$. We have $\operatorname{val}_{\mathcal{S}}(w \ell)=n$. Let $m=\operatorname{val}_{\mathcal{S}}(w)$. Since $w \in L \backslash 0 C^{*}$, then $\operatorname{rep}_{\mathcal{S}}(m)=w$. From [BR10, Corollary 3.4.14], we have

$$
\sigma\left(x_{\mathrm{val}_{\mathcal{S}}(w)}\right)=x_{\mathrm{val}_{\mathcal{S}}(w 0)} x_{\mathrm{val}_{\mathcal{S}}(w 1)} \cdots x_{\mathrm{val}_{\mathcal{S}}(w \ell)} \cdots x_{\mathrm{val}_{\mathcal{S}}(w \cdot(K-1))}
$$

where $K=\left|\sigma\left(x_{\operatorname{val}_{\mathcal{S}}(w)}\right)\right|$. Thus $x_{n}=x_{\mathrm{val}_{\mathcal{S}}(w \ell)}=\sigma\left(x_{\mathrm{val}_{\mathcal{S}}(w)}\right)[\ell]=\sigma\left(x_{m}\right)[\ell]$. The other statement follows from the equation [BR10, (3.12)].

Let $\varphi$ be the morphism $\varphi: a \mapsto a b, b \mapsto a$. The automaton $\mathcal{A}_{\varphi^{2}, a}$ associated to the right-infinite fixed point of $\varphi^{2}$ starting with letter $a$ is shown in Figure 4. We construct another automaton $\mathcal{A}_{\varphi^{2}, s}$ associated to the bi-infinite fixed point of $\varphi^{2}$ defined from the seed $s=b . a$, see Figure 4 . The bi-infinite Fibonacci word is $x=\lim _{k \rightarrow+\infty} \varphi^{2 k}(b . a)$, where the dot represents the origin between positions -1 and 0 . When referring to $\varphi^{2 k}(b$. $)$ we mean the finite word $\varphi^{2 k}(b)=$ $x_{-\left|\varphi^{2 k}(b)\right|} \ldots x_{-2} x_{-1}$.
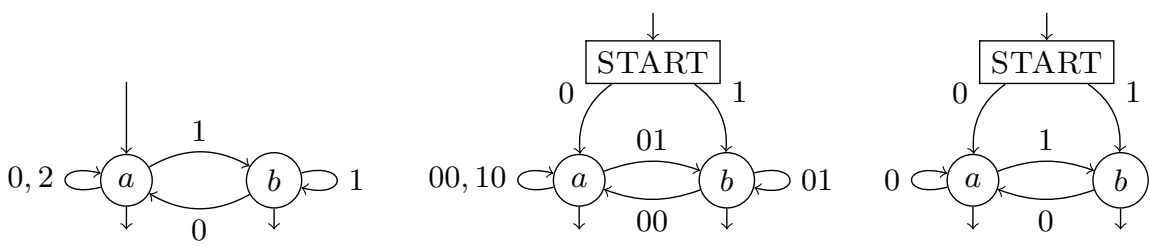

Fig. 4. Automata $\mathcal{A}_{\varphi^{2}, a}, \mathcal{A}_{\varphi^{2}, s}$ and $\mathcal{A}_{\varphi, s}$ with seed $s=b . a$.

Lemma 8. Let $x=\lim _{k \rightarrow+\infty} \varphi^{2 k}($ b.a $)$ and let $n \in \mathbb{Z} \backslash I_{0}$. Then there exist integers $m \in \mathbb{Z}$ and $0 \leq \ell<3$ such that

$$
x[n]=\varphi^{2}(x[m])[\ell], \text { with } \operatorname{rep}_{\mathcal{F}}(n)=\operatorname{rep}_{\mathcal{F}}(m) h(\ell),
$$


where $h$ is the morphism $h:\{0,1,2\}^{*} \rightarrow\{0,1\}^{*}$ defined as $h: 0 \mapsto 00,1 \mapsto$ $01,2 \mapsto 10$. Moreover, if $n \in I_{i} \backslash I_{i-1}$ for some $1 \leq i \leq k$, then $m \in I_{i-1} \backslash I_{i-2}$.

Proof. First assume that $n>0$. Let $\mathcal{A}_{\varphi^{2}, a}$ and $\mathcal{G}=\left(L \backslash 0 C^{*}, C,<\right)$. Then $x=$ $\lim _{k \rightarrow+\infty} \varphi^{2 k}(a)$ is $\mathcal{G}$-automatic, i.e., for any $n \in \mathbb{N}$, we have $x_{n}=\mathcal{A}_{\varphi^{2}, a}\left(\operatorname{rep}_{\mathcal{G}}(n)\right)$, where $\operatorname{rep}_{\mathcal{G}}(n) \in\{\varepsilon, 1,2,10,11,20,21,22,100,101, \ldots\}$. From the definition of $\mathcal{A}_{\varphi^{2}, s}$ in Figure 4, we have $x_{n}=\mathcal{A}_{\varphi^{2}, s}\left(0 h\left(\operatorname{rep}_{\mathcal{G}}(n)\right)\right)$. Moreover, from Proposition 7 , for $n>0$ there exist integers $m \in \mathbb{N}$ and $\ell$ such that $h\left(\operatorname{rep}_{\mathcal{G}}(n)\right)=$ $h\left(\operatorname{rep}_{\mathcal{G}}(m)\right) h(l)$. On the other hand, $0 h\left(\operatorname{rep}_{\mathcal{G}}(n)\right)=\operatorname{rep}_{\mathcal{F}}(n)$ for all $n \in \mathbb{N}$. This follows from the following reasons. Applying Proposition 7, we obtain that $\left|0 h\left(\operatorname{rep}_{\mathcal{G}}(n)\right)\right|=2 i+1$ if and only if $n \in\left(I_{i} \backslash I_{i-1}\right) \cap \mathbb{N}$. The alphabet $h(C)$ has the same ordering as the alphabet $C$. Finally, a word 12 is not accepted by the automaton $\mathcal{A}_{\varphi^{2}, a}$ and therefore a word 11 is forbidden in $0 h(L)$. Moreover, as $|h(\ell)|=2$, we observe $\left|\operatorname{rep}_{\mathcal{F}}(m)\right|=\left|\operatorname{rep}_{\mathcal{F}}(n)\right|-2$, thus $m \in I_{i-1} \backslash I_{i-2}$.

If $n=-2$, then we denote $\ell=0, m=-1$. Let $n<-2$ and let $k \geq 2$ be such that $n \in I_{k} \backslash I_{k-1}$. We have

$$
\varphi^{2 k}(b .)[n]=\varphi^{2 k-1}(a)\left[n+\left|\varphi^{2 k}(b)\right|\right],
$$

where $\left|\varphi^{2 k}(b)\right|=F_{2 k}$. As $\varphi^{2 k-1}(a)$ is a prefix of $\varphi^{2 k}(a)$, we can write

$$
\varphi^{2 k-1}(a)\left[n+F_{2 k}\right]=\varphi^{2 k}(a)\left[n+F_{2 k}\right] .
$$

Then $0 \leq n+F_{2 k}<F_{2 k-1}$ and we denote $0 \leq i \leq k-1$ such that $n+F_{2 k} \in I_{i} \backslash I_{i-1}$. In case that $i>0$, we use the previous paragraph for positive $n>0$ on a long enough prefix $z$ of $x\left(z=\varphi^{2 k-2}(a)\right.$, therefore $\left.n+F_{2 k}<|z|=F_{2 k-1}\right)$ and we find $m_{P} \in I_{i-1} \backslash I_{i-2}$ and $\ell$ such that $\operatorname{rep}_{\mathcal{F}}\left(n+F_{2 k}\right)=\operatorname{rep}_{\mathcal{F}}\left(m_{P}\right) h(\ell)$ and

$$
\varphi^{2}\left(\varphi^{2 k-2}(a)\right)\left[n+F_{2 k}\right]=\varphi^{2}\left(\varphi^{2 k-2}(a)\left[m_{P}\right]\right)[\ell] .
$$

As $0 \leq m_{P}<F_{2 k-3}<F_{2 k-1}=\left|\varphi^{2 k-2}(a)\right|$, we restrict the last relation just to a prefix $\varphi^{2 k-3}(a)$ and use the relation (1) again to get $m=m_{P}-F_{2 k-2}<0$

$$
\varphi^{2 k}(b .)[n]=\varphi^{2}\left(\varphi^{2 k-3}(a)\left[m_{P}\right]\right)[\ell]=\varphi^{2}\left(\varphi^{2 k-2}(b .)\left[m_{P}-F_{2 k-2}\right]\right)[\ell] .
$$

The representation $\operatorname{rep}_{\mathcal{F}}(n)=10 \mathrm{w}$ has the first digit corresponding to $-F_{2 k}$. Then $(00)^{k-i} \operatorname{rep}_{\mathcal{F}}\left(n+F_{2 k}\right)=00 w$. As $m_{P} \in I_{i-1} \backslash I_{i-2}$, then $m_{P}-F_{2 k-2} \in I_{k-1} \backslash I_{k-2}$ and $\operatorname{rep}_{\mathcal{F}}\left(m_{P}-F_{2 k-2}\right)=10(00)^{k-i-1} \operatorname{rep}_{\mathcal{F}}\left(m_{P}\right)$. As a whole,

$\operatorname{rep}_{\mathcal{F}}(n)=10(00)^{k-i-1} \operatorname{rep}_{\mathcal{F}}\left(m_{P}\right) h(\ell)=\operatorname{rep}_{\mathcal{F}}\left(m_{P}-F_{2 k-2}\right) h(\ell)=\operatorname{rep}_{\mathcal{F}}(m) h(\ell)$.

If $i=0$, then we denote $\ell=0, m=-F_{2 k-2}$ and the statement holds true.

We show the following result.

Proposition 9. The DFAO $\mathcal{A}_{\varphi, s}$ associated to the seed $s=b . a$ satisfies

$$
x_{n}=\mathcal{A}_{\varphi, s}\left(\operatorname{rep}_{\mathcal{F}}(n)\right) \text { for all } n \in \mathbb{Z} .
$$


Proof. Let $\mathcal{A}_{\varphi, s}$ be the automaton shown in Figure 4, i.e., the DFAO $\mathcal{A}_{\varphi, s}=$ $(\{a, b\} \cup\{\operatorname{START}\},\{0,1\}, \delta$, START, $\{a, b\})$ with the partial function $\delta$ such that

$-\delta\left(\operatorname{START}, \operatorname{rep}_{\mathcal{F}}(n)\right)=s_{n}$ for every $n \in I_{0}=\{-1,0\}$, where $s_{-1}=b, s_{0}=a$,

$-\delta(c, i)=d$ for any $c, d \in\{a, b\}$ if and only if $\varphi(c)=u$ and $u_{i}=d$.

Assume $n \in I_{0}$. If $n=0$, then we have $x_{0}=a=\mathcal{A}_{\varphi, s}(0)=\mathcal{A}_{\varphi, s}\left(\operatorname{rep}_{\mathcal{F}}(0)\right)$. If $n=-1$, then $x_{-1}=b=\mathcal{A}_{\varphi, s}(1)=\mathcal{A}_{\varphi, s}\left(\operatorname{rep}_{\mathcal{F}}(-1)\right)$. Induction hypothesis: we assume for some $k \in \mathbb{N}$ that $x_{m}=\mathcal{A}_{\varphi, s}\left(\operatorname{rep}_{\mathcal{F}}(m)\right)$ for all $m \in I_{k} \backslash I_{k-1}$. Let $n \in I_{k+1} \backslash I_{k}$. Then from Lemma 8 there exist $m \in I_{k} \backslash I_{k-1}$ and $\ell \in\{0,1,2\}$ such that $x_{n}=\varphi^{2}\left(x_{m}\right)[\ell]$ and $\operatorname{rep}_{\mathcal{F}}(n)=\operatorname{rep}_{\mathcal{F}}(m) \cdot h(\ell)$, where $h(\ell)=\ell_{0} \cdot \ell_{1}$ for some $\ell_{0}, \ell_{1} \in\{0,1\}$. From the induction hypothesis, we have

$$
\begin{aligned}
x_{n} & =\varphi^{2}\left(x_{m}\right)[\ell]=\varphi^{2}\left(\mathcal{A}_{\varphi, s}\left(\operatorname{rep}_{\mathcal{F}}(m)\right)\right)[\ell]=\varphi\left(\varphi\left(\mathcal{A}_{\varphi, s}\left(\operatorname{rep}_{\mathcal{F}}(m)\right)\right)\left[\ell_{0}\right]\right)\left[\ell_{1}\right] \\
& =\mathcal{A}_{\varphi, s}\left(\operatorname{rep}_{\mathcal{F}}(m) \cdot \ell_{0} \cdot \ell_{1}\right)=\mathcal{A}_{\varphi, s}\left(\operatorname{rep}_{\mathcal{F}}(m) h(\ell)\right)=\mathcal{A}_{\varphi, s}\left(\operatorname{rep}_{\mathcal{F}}(n)\right) .
\end{aligned}
$$

\section{Two-dimensional Words, Languages and Morphisms}

In this section, we introduce 2-dimensional words, languages and morphisms following the notations of [CKR10,Lab21]. Let $k \in \mathbb{N}$ and $\mathcal{A}=\{0,1, \ldots, k\}$ be a finite alphabet and let $u:\left\{0, \ldots, n_{1}-1\right\} \times\left\{0, \ldots, n_{2}-1\right\} \rightarrow \mathcal{A}$ be a $2-$ dimensional word of shape $\boldsymbol{n}=\left(n_{1}, n_{2}\right) \in \mathbb{N}^{2}$. Let $\mathcal{A}^{\boldsymbol{n}}$ denote the set of all 2 -dimensional words of shape $\boldsymbol{n}$. We refer to the words of shape $(1,2),(2,1)$ as to the vertical, horizontal dominoes, respectively. We represent a 2-dimensional word $u$ of shape $\left(n_{1}, n_{2}\right) \in \mathbb{N}^{2}$ as a matrix with Cartesian coordinates:

$$
u=\left(\begin{array}{ccc}
u_{0, n_{2}-1} \ldots & u_{n_{1}-1, n_{2}-1} \\
\ldots & \ldots & \ldots \\
u_{0,0} & \ldots & u_{n_{1}-1,0}
\end{array}\right) .
$$

Let $\mathcal{A}^{* 2}=\bigcup_{\mathbf{n} \in \mathbb{N}^{2}} \mathcal{A}^{\mathbf{n}}$ the set of all 2-dimensional words. Let $u, v \in \mathcal{A}^{* 2}$ be of shape $\left(n_{1}, n_{2}\right),\left(\tilde{n}_{1}, \tilde{n}_{2}\right)$, respectively. If $n_{2}=\tilde{n}_{2}$, the concatenation in direction $e_{1}$ is defined as a 2-dimensional word $u \odot{ }^{1} v$ of shape $\left(n_{1}+\tilde{n}_{1}, n_{2}\right)$ given as

$$
u \odot^{1} v=\left(\begin{array}{cccccc}
u_{0, n_{2}-1} \ldots & u_{n_{1}-1, n_{2}-1} & v_{0, n_{2}-1} \ldots \ldots & v_{\tilde{n_{1}}-1, n_{2}-1} \\
\ldots & \ldots & \ldots & \ldots & \ldots & \ldots \\
u_{0,0} & \ldots & u_{n_{1}-1,0} & v_{0,0} & \ldots & v_{\tilde{n}_{1}-1,0}
\end{array}\right) .
$$

If $n_{1}=\tilde{n}_{1}$, the concatenation in direction $\boldsymbol{e}_{2}$ is defined analogically. A word $v \in \mathcal{A}^{* 2}$ is a subword of a word $u \in \mathcal{A}^{* 2}$ if there exist words $u_{1}, u_{2}, u_{3}, u_{4} \in \mathcal{A}^{* 2}$ such that $u=u_{3} \odot^{2}\left(u_{1} \odot^{1} v \odot^{1} u_{2}\right) \odot^{2} u_{4}$.

A subset $L \subseteq \mathcal{A}^{* 2}$ is called a 2-dimensional factorial language if $u \in L$ implies that $v \in L$ for all 2-dimensional subwords $v$ of $u$.

Let $\mathcal{A}$ and $\mathcal{B}$ be two alphabets. Let $L \subseteq \mathcal{A}^{*^{2}}$ be a factorial language. A function $\omega: L \rightarrow \mathcal{B}^{*^{2}}$ is a 2-dimensional morphism if for every $i$ with $1 \leq i \leq 2$, 
and every $u, v \in L$ such that $u \odot^{i} v$ is defined and $u \odot^{i} v \in L$, we have that the concatenation $\omega(u) \odot^{i} \omega(v)$ in direction $\boldsymbol{e}_{i}$ is defined and

$$
\omega\left(u \odot^{i} v\right)=\omega(u) \odot^{i} \omega(v) .
$$

A 2-dimensional morphism $L \rightarrow \mathcal{B}^{*^{2}}$ is thus completely defined from the image of the letters in $\mathcal{A}$ and can be denoted as a rule $\mathcal{A} \rightarrow \mathcal{B}^{*^{2}}$.

A subset $X \subseteq \mathcal{A}^{\mathbb{Z}^{2}}$ is called a subshift if it is closed under the $\operatorname{shift}^{5} \sigma$ and closed with respect to compact product topology. Let $L \subseteq \mathcal{A}^{*^{2}}$ be a factorial language and $\mathcal{L}(x)$ be the factorial language containing all subwords of the configuration $x \in \mathcal{A}^{\mathbb{Z}^{2}}$. Then, $\mathcal{X}_{L}=\left\{x \in \mathcal{A}^{\mathbb{Z}^{2}} \mid \mathcal{L}(x) \subset L\right\}$ is a subshift generated by $L$. A 2-dimensional morphism $\omega: L \rightarrow \mathcal{B}^{*^{2}}$ can be extended to a continuous map $\omega: \mathcal{X}_{L} \rightarrow \mathcal{B}^{\mathbb{Z}^{2}}$ in such a way that the origin of $\omega(x)$ is at zero position in the word $\omega\left(x_{(0,0)}\right)$ for all $x \in \mathcal{X}_{L}$.

In general, the closure under the shift of the image of a subshift $X \subseteq \mathcal{A}^{\mathbb{Z}^{2}}$ under $\omega$ is the subshift $\overline{\omega(X)}{ }^{\sigma}=\left\{\sigma^{k} \omega(x) \in \mathcal{B}^{\mathbb{Z}^{2}} \mid \boldsymbol{k} \in \mathbb{Z}^{2}, x \in X\right\} \subseteq \mathcal{B}^{\mathbb{Z}^{2}}$.

A 2-dimensional morphism $\omega: \mathcal{A} \rightarrow \mathcal{A}^{*^{2}}$ is said expansive if the width and height of $\omega^{k}(a)$ goes to $\infty$ for all letters $a \in \mathcal{A}$. A subshift $X \subset \mathcal{A}^{\mathbb{Z}^{2}}$ is selfsimilar if there exists an expansive 2-dimensional morphism $\mathcal{A} \rightarrow \mathcal{A}^{*^{2}}$ such that $X=\overline{\omega(X)}{ }^{\sigma}$.

\section{Self-similarity of the Wang Shift $\Omega_{\mathcal{Z}}$}

Proposition 10. The Wang shift $\Omega_{\mathcal{Z}}$ is self-similar satisfying ${\overline{\phi\left(\Omega_{\mathcal{Z}}\right)}}^{\sigma}=\Omega_{\mathcal{Z}}$ where $\phi$ is the 2-dimensional morphism over the alphabet $\mathcal{H}=\{0, \ldots, 15\}$

$$
\begin{aligned}
& \phi: \mathcal{H} \rightarrow \mathcal{H}^{*^{2}}
\end{aligned}
$$

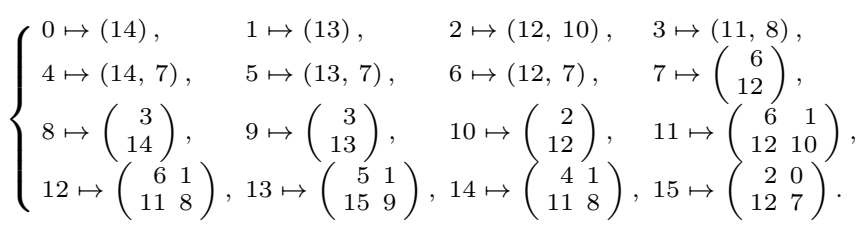

The proof of Proposition 10 is done in the appendix. It is using algorithms to desubstitute Wang shifts based on the notion of marker tiles [Lab21].

Similarly to the 1-dimensional case, we can build an automaton associated to a fixed point of the 2-dimensional morphism $\phi$ defined in Equation (2). Let $s=\left(\begin{array}{cc}8 & 12 \\ 1 & 6\end{array}\right) \in \mathcal{H}^{(2,2)}$ be the seed associating one letter to each quadrant. We observe that $\phi^{2}(s)$ prolongates $s$ at the origin. Therefore, $\lim _{k \rightarrow \infty} \phi^{2 k}(s)$ defines a configuration in $\mathcal{H}^{\mathbb{Z}^{2}}$ which is a fixed point of $\phi^{2}$.

Associated to the morphism $\phi$ and to the seed $s=\left(\begin{array}{cc}s_{(-1,0)} & s_{(0,0)} \\ s_{(-1,-1)} & s_{(0,-1)}\end{array}\right) \in$ $\mathcal{H}^{(2,2)}$, we construct a DFAO $\mathcal{A}_{\phi, s}=(\mathcal{H} \cup\{\operatorname{START}\}, \Sigma, \delta, I, \mathcal{H})$ such that $\Sigma=$ $\left\{\left(\begin{array}{l}0 \\ 0\end{array}\right),\left(\begin{array}{l}0 \\ 1\end{array}\right),\left(\begin{array}{l}1 \\ 0\end{array}\right),\left(\begin{array}{l}1 \\ 1\end{array}\right)\right\}, I=\{$ START $\}$ and $\delta: Q \times \Sigma \rightarrow Q$ is a partial function such that

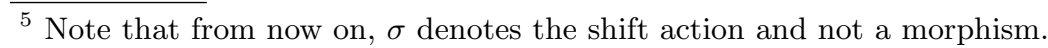


$-\delta\left(\operatorname{START}, \operatorname{rep}_{\mathcal{F}}(\boldsymbol{n})\right)=s_{\boldsymbol{n}}$ for every $\boldsymbol{n} \in I_{0}^{2}=\{(0,0),(-1,0),(0,-1),(-1,-1)\}$,

- $\delta(a, e)=b$ for any $a, b \in \mathcal{H}$ and $e \in \Sigma$ if and only if $b$ is in $\phi(a)$ at position $e$.

The automaton $\mathcal{A}_{\phi, s}$ associated to the morphism $\phi$ and seed $s=\left(\begin{array}{ll}8 & 12 \\ 1 & 6\end{array}\right)$ is shown in Figure 5.

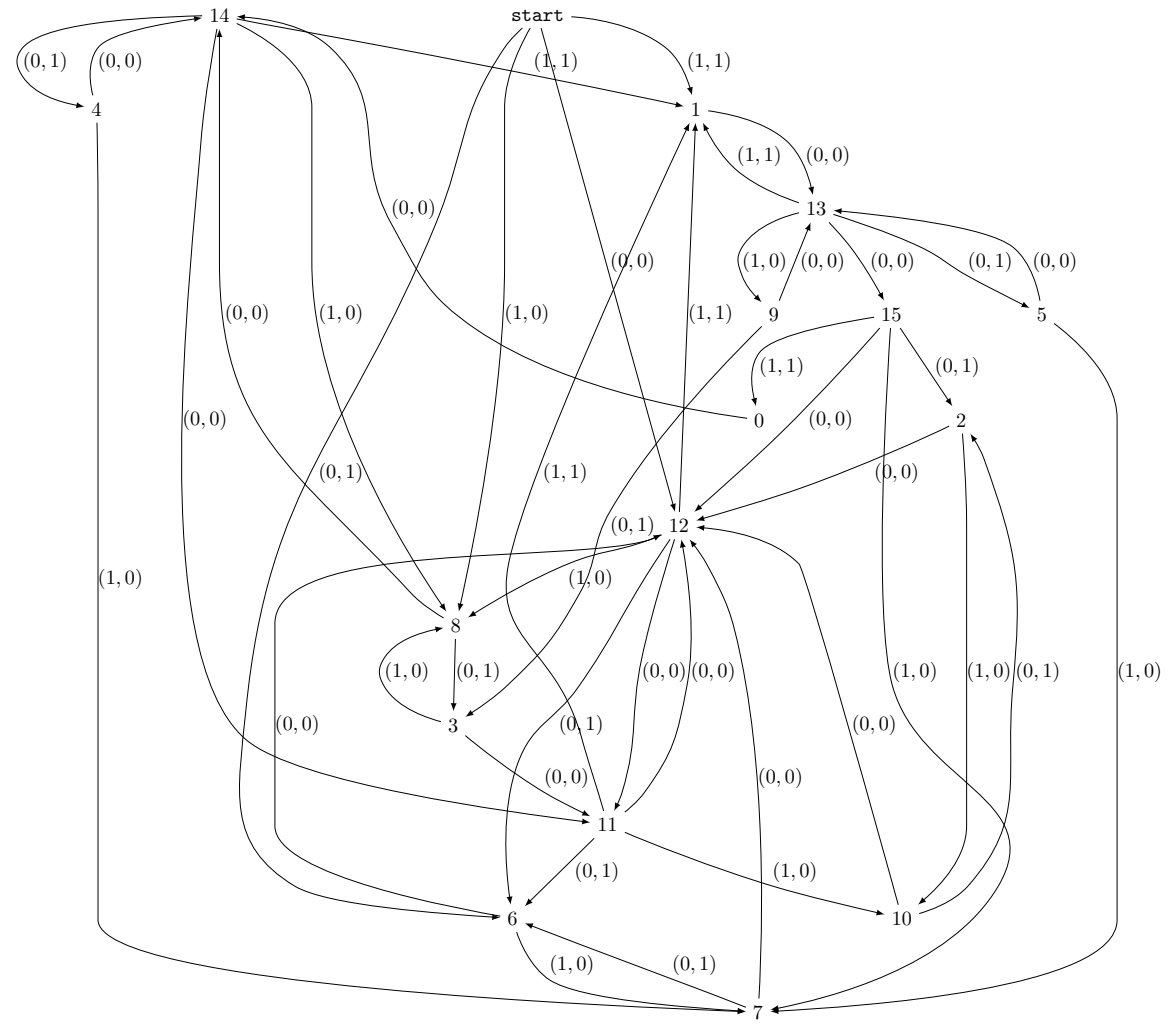

Fig. 5. The automaton $\mathcal{A}_{\phi, s}$ associated to the 2-dimensional morphism $\phi$ and seed $s=\left(\begin{array}{cc}8 & 12 \\ 1 & 6\end{array}\right)$.

\section{Proof of Main Results}

In this section, we prove Theorem 1. The strategy is to extract the horizontal and vertical structure (expressed as 1-dimensional morphisms) of a 2-dimensional morphism and exploit results proved for the 1-dimensional case in Section 3, in particular Lemma 8. 
Let $\omega$ be a 2-dimensional morphism on the alphabet $\mathcal{Q}$ and $\mathcal{X}_{\omega}$ be the associated substitutive subshift. Since $\omega: \mathcal{X}_{\omega} \rightarrow \mathcal{X}_{\omega}$ is well-defined, it imposes that the horizontal width of $\omega(a)$ equals the horizontal width of $\omega(b)$ for every pair of letters $a, b \in \mathcal{Q}$ appearing in the same column. This holds also for the height of the images of letters appearing in the same row. However, more can be said.

We define $\sim_{\text {col }}$ the equivalence relation as the reflexive, symmetric and transitive closure of the relation $\left\{(a, b) \mid\left(\begin{array}{l}b \\ a\end{array}\right) \in \mathcal{L}_{\omega}\right\}$ made of the vertical dominoes in the language. We define $\sim_{\text {row }}$ the equivalence relation as the reflexive, symmetric and transitive closure of the relation $\left\{(a, b) \mid(a b) \in \mathcal{L}_{\omega}\right\}$ made of the horizontal dominoes in the language. We have that $a \sim_{\text {col }} b$ if and only if letters $a$ and $b$ appear in the same column in some configuration of $\mathcal{X}_{\omega}$. Let $\pi_{\text {col }}:\left.\mathcal{Q} \rightarrow \mathcal{Q}\right|_{\sim_{\text {col }}}$ and $\pi_{\text {row }}:\left.\mathcal{Q} \rightarrow \mathcal{Q}\right|_{\sim_{\text {row }}}$ be the maps defined as $\pi_{\text {col }}: a \mapsto[a]_{\sim_{\text {col }}}$, and $\pi_{\text {row }}: a \mapsto[a]_{\sim_{\text {row }}}$ mapping a letter $a \in \mathcal{Q}$ to its equivalence class.

We define the horizontal and vertical structure of $\omega$ resp. as the 1-dimensional morphisms $\omega_{\text {HORIZ }}:\left.\left.\mathcal{Q}\right|_{\sim_{\text {col }}} ^{*} \rightarrow \mathcal{Q}\right|_{\sim_{\text {col }}} ^{*}$ and $\omega_{\text {VERT }}:\left.\left.\mathcal{Q}\right|_{\sim_{\text {row }}} ^{*} \rightarrow \mathcal{Q}\right|_{\sim_{\text {row }}} ^{*}$ by

where $w=\omega(a) \in \mathcal{Q}^{(m, n)}$.

$$
\begin{aligned}
& \omega_{\text {HORIZ }}\left([a]_{\sim_{\text {col }}}\right)=\left[w_{0,0}\right]_{\sim_{\text {col }}} \cdots\left[w_{m-1,0}\right]_{\sim_{\text {col }}}, \\
& \omega_{\text {VERT }}\left([a]_{\sim_{\text {row }}}\right)=\left[w_{0,0}\right]_{\sim_{\text {row }}} \cdots\left[w_{0, n-1}\right]_{\sim_{\text {row }}}
\end{aligned}
$$

Lemma 11. Let $\phi$ be the 2-dimensional morphism from Proposition 10 and $x$ the point fixed by $\phi^{2}, x=\lim _{k \rightarrow+\infty} \phi^{2 k}(s)$ for the seed $s=\left(\begin{array}{cc}8 & 12 \\ 1 & 6\end{array}\right)$. Let $\boldsymbol{n} \in \mathbb{Z}^{2} \backslash I_{0}^{2}$. Then, there exist vectors $\boldsymbol{m} \in \mathbb{Z}^{2}$ and $\boldsymbol{\ell} \in\{0,1,2\}^{2}$ such that

$$
x_{\boldsymbol{n}}=\phi^{2}\left(x_{\boldsymbol{m}}\right)[\boldsymbol{\ell}] \text {, where } \operatorname{rep}_{\mathcal{F}}(\boldsymbol{n})=\operatorname{rep}_{\mathcal{F}}(\boldsymbol{m}) \cdot h(\boldsymbol{\ell}) \text {. }
$$

Proof. Let $\boldsymbol{n} \in \mathbb{Z}^{2} \backslash I_{0}^{2}$. The powers $\phi^{2 k}(s)$ are defined such that they grow in all four quadrants. The vertical and horizontal structure of $\phi$ are respectively

$$
\begin{aligned}
\phi_{\mathrm{VERT}}=\phi_{\mathrm{HORIZ}}= & \left\{\begin{array}{l}
a \mapsto a b \\
b \mapsto a
\end{array}\right. \text { satisfying } \\
& \operatorname{width}(\phi(w))=\left|\phi_{\mathrm{HORIZ}} \circ \pi_{\text {col }}\left(w_{0,0} \cdots w_{m-1,0}\right)\right| \\
& \operatorname{height}(\phi(w))=\left|\phi_{\mathrm{VERT}} \circ \pi_{\text {row }}\left(w_{0,0} \cdots w_{0, n-1}\right)\right|
\end{aligned}
$$

for all 2-dimensional words $w \in \mathcal{L}_{\phi}$ of shape $(m, n)$. Therefore, the vectors $\boldsymbol{m}$ and $\ell$ we are searching for can be found coordinate by coordinate.

Let $y=\lim _{k \rightarrow \infty} \phi_{\mathrm{HORIZ}}^{2 k}(b . a)=\lim _{k \rightarrow \infty} \phi_{\mathrm{VERT}}^{2 k}(b . a)$. From Lemma 8 , there exist integers $m_{1}, m_{2} \in \mathbb{Z}$ and $0 \leq \ell_{1}, \ell_{2}<3$ such that

$$
y\left[n_{1}\right]=\phi_{\mathrm{HORIZ}}^{2}\left(y\left[m_{1}\right]\right)\left[\ell_{1}\right] \quad \text { and } \quad y\left[n_{2}\right]=\phi_{\mathrm{VERT}}^{2}\left(y\left[m_{2}\right]\right)\left[\ell_{2}\right],
$$

where $\operatorname{rep}_{\mathcal{F}}\left(n_{1}\right)=\operatorname{rep}_{\mathcal{F}}\left(m_{1}\right) \cdot h\left(\ell_{1}\right)$ and $\operatorname{rep}_{\mathcal{F}}\left(n_{2}\right)=\operatorname{rep}_{\mathcal{F}}\left(m_{2}\right) \cdot h\left(\ell_{2}\right)$. Moreover, it satisfies $x_{\left(n_{1}, n_{2}\right)}=\phi^{2}\left(x_{\left(m_{1}, m_{2}\right)}\right)\left[\left(\ell_{1}, \ell_{2}\right)\right]$. We conclude

$\operatorname{rep}_{\mathcal{G}}(\boldsymbol{n})=\left(\begin{array}{l}\operatorname{pad}_{t}\left(\operatorname{rep}_{\mathcal{F}}\left(n_{1}\right)\right) \\ \operatorname{pad}_{t}\left(\operatorname{rep}_{\mathcal{F}}\left(n_{2}\right)\right)\end{array}\right)=\left(\begin{array}{l}\operatorname{pad}_{t-2}\left(\operatorname{rep}_{\mathcal{F}}\left(m_{1}\right) \cdot h\left(\ell_{1}\right)\right. \\ \operatorname{pad}_{t-2}\left(\operatorname{rep}_{\mathcal{F}}\left(m_{2}\right) \cdot h\left(\ell_{2}\right)\right.\end{array}\right)=\operatorname{rep}_{\mathcal{F}}(\boldsymbol{m}) \cdot h(\ell)$.

where $t=\max \left\{\left|\operatorname{rep}_{\mathcal{F}}\left(n_{1}\right)\right|,\left|\operatorname{rep}_{\mathcal{F}}\left(n_{2}\right)\right|\right\}$. 
Lemma 12. For any state $r \in Q \backslash\{\mathrm{START}\}$ in the automaton $\mathcal{A}_{\phi, s}$ and any $\ell \in\left\{0, \ldots, \operatorname{width}\left(\phi^{2}(r)\right)-1\right\} \times\left\{0, \ldots\right.$, height $\left.\left(\phi^{2}(r)\right)-1\right\}$ we have

$$
\mathcal{A}_{\phi, s}(r, h(\ell))=\phi^{2}(r)[\ell] .
$$

Proof. Let $r$ and $\boldsymbol{\ell}$ be according to the assumptions. Therefore, $\boldsymbol{\ell} \in\{0,1,2\}^{2}$. Then, there exist unique vectors $\boldsymbol{\ell}_{0}, \boldsymbol{\ell}_{1} \in\{0,1\}^{2}$ such that $\phi^{2}(s)[\ell]=\phi\left(\phi(s)\left[\boldsymbol{\ell}_{0}\right]\right)\left[\boldsymbol{\ell}_{1}\right]$. As $\boldsymbol{\ell}_{0}, \boldsymbol{\ell}_{1} \in\{0,1\}^{2}$, they belong to the set of edges $\Sigma$ of the automaton $\mathcal{A}_{\phi, s}$. Then, using the general properties of an automaton we have

$$
\begin{aligned}
\phi^{2}(r)[\boldsymbol{\ell}] & =\phi\left(\phi(r)\left[\boldsymbol{\ell}_{0}\right]\right)\left[\boldsymbol{\ell}_{1}\right]=\phi\left(\mathcal{A}_{\phi, s}\left(r, \boldsymbol{\ell}_{0}\right)\right)\left[\boldsymbol{\ell}_{1}\right] \\
& =\mathcal{A}_{\phi, s}\left(\mathcal{A}_{\phi, s}\left(r, \boldsymbol{\ell}_{0}\right), \boldsymbol{\ell}_{1}\right)=\mathcal{A}_{\phi, s}\left(r, \boldsymbol{\ell}_{0} \boldsymbol{\ell}_{1}\right)=\mathcal{A}_{\phi, s}(r, h(\boldsymbol{\ell})),
\end{aligned}
$$

where the last equation holds for the following reasons.

I) If $\boldsymbol{\ell} \in\{0,1\}^{2},\left(\begin{array}{c}i \\ j\end{array}\right)=\boldsymbol{\ell}$, then $h(\boldsymbol{\ell})=\left(\begin{array}{ll}0 & i \\ 0 & j\end{array}\right)$. Also, $\boldsymbol{\ell}_{0}=\left(\begin{array}{l}0 \\ 0\end{array}\right)$ and $\boldsymbol{\ell}_{1}=\left(\begin{array}{l}i \\ j\end{array}\right)$.

II) If $\ell \in\{2\} \times\{0,1\},\left(\begin{array}{l}2 \\ j\end{array}\right)=\boldsymbol{\ell}$, then $h(\ell)=\left(\begin{array}{ll}1 & 0 \\ 0 & j\end{array}\right)$. On the other hand, $\boldsymbol{\ell}_{0}=\left(\begin{array}{l}1 \\ 0\end{array}\right)$ and $\boldsymbol{\ell}_{1}=\left(\begin{array}{l}0 \\ j\end{array}\right)$. The case $\boldsymbol{\ell} \in\{0,1\} \times\{2\}$ is analogical.

III) If $\boldsymbol{\ell} \in\{2\}^{2}$, then $h(\boldsymbol{\ell})=\left(\begin{array}{ll}1 & 0 \\ 1 & 0\end{array}\right)$. Also, $\boldsymbol{\ell}_{0}=\left(\begin{array}{l}1 \\ 1\end{array}\right)$ and $\boldsymbol{\ell}_{1}=\left(\begin{array}{l}0 \\ 0\end{array}\right)$.

Theorem 13. Let $\phi$ be a 2-dimensional morphism and $x$ the point fixed by $\phi^{2}$, $x=\lim _{k \rightarrow+\infty} \phi^{2 k}(s)$ for a seed $s=\left(\begin{array}{cc}8 & 12 \\ 1 & 6\end{array}\right)$. Then, there exists an automaton $\mathcal{A}$ such that $x_{\boldsymbol{n}}=\mathcal{A}\left(\operatorname{rep}_{\mathcal{F}}(\boldsymbol{n})\right)$.

Proof. Let $\mathcal{A}=\mathcal{A}_{\phi, s}$ the automaton associated to the morphism $\phi$ and seed $s=$ $\left(\begin{array}{cc}8 & 12 \\ 1 & 6\end{array}\right)$. If $\boldsymbol{n} \in I_{0}^{2}=\{(0,0),(-1,0),(-1,-1),(0,-1)\}$, then $x_{\boldsymbol{n}}=\mathcal{A}\left(\operatorname{rep}_{\mathcal{F}}(\boldsymbol{n})\right)$. Induction hypothesis: we assume for some $k \in \mathbb{N}$ that $x_{\boldsymbol{m}}=\mathcal{A}\left(\operatorname{rep}_{\mathcal{F}}(\boldsymbol{m})\right)$ for all $\boldsymbol{m} \in I_{k}^{2} \backslash I_{k-1}^{2}$. Let $\boldsymbol{n} \in I_{k+1}^{2} \backslash I_{k}^{2}$. Then, from Lemma 11 there exist $\boldsymbol{m} \in \mathbb{Z}^{2}$ and $\boldsymbol{\ell} \in\{0,1,2\}^{2}$ such that $x_{\boldsymbol{n}}=\phi^{2}\left(x_{\boldsymbol{m}}\right)[\boldsymbol{\ell}]$ where $\operatorname{rep}_{\mathcal{F}}(\boldsymbol{n})=\operatorname{rep}_{\mathcal{F}}(\boldsymbol{m}) \cdot h(\boldsymbol{\ell})$.

This implies $\left|\operatorname{rep}_{\mathcal{F}}(\boldsymbol{m})\right|=\left|\operatorname{rep}_{\mathcal{F}}(\boldsymbol{n})\right|-2$, and therefore by Lemma $6, \boldsymbol{m} \in$ $I_{k}^{2} \backslash I_{k-1}^{2}$. From the induction hypothesis, $x_{\boldsymbol{m}}=\mathcal{A}\left(\operatorname{rep}_{\mathcal{F}}(\boldsymbol{m})\right)$. Then, from the induction hypothesis and Lemma 12, we have

$$
\begin{aligned}
x_{\boldsymbol{n}} & =\phi^{2}\left(x_{\boldsymbol{m}}\right)[\boldsymbol{\ell}]=\phi^{2}\left(\mathcal{A}\left(\operatorname{rep}_{\mathcal{F}}(\boldsymbol{m})\right)\right)[\boldsymbol{\ell}] \\
& =\mathcal{A}\left(\operatorname{rep}_{\mathcal{F}}(\boldsymbol{m}) h(\boldsymbol{\ell})\right)=\mathcal{A}\left(\operatorname{rep}_{\mathcal{F}}(\boldsymbol{n})\right) .
\end{aligned}
$$

Proof (of Theorem 1). Let $\phi$ be the 2-dimensional morphism from Proposition 10 and let $x=\phi^{2}(x)$ be the point fixed by $\phi^{2}$, where $x=\lim _{k \rightarrow+\infty} \phi^{2 k}(s)$ of the seed $s=\left(\begin{array}{ll}8 & 12 \\ 1 & 6\end{array}\right)$. Let $\mathcal{A}=\mathcal{A}_{\phi, s}$ (see Figure 5 ). The conclusion follows from Theorem 13 .

Example 14. Let $\boldsymbol{n}=(-1,6) \in \mathbb{Z}^{2}$. Then, $\operatorname{rep}_{\mathcal{F}}(\boldsymbol{n})=\left(\begin{array}{c}10101 \\ 01001\end{array}\right)$ and $\mathcal{A}_{\phi, s}$ gives

$$
\text { START } \stackrel{(1,0)}{\longrightarrow} 8 \stackrel{(0,1)}{\longrightarrow} 3 \stackrel{(1,0)}{\longrightarrow} 8 \stackrel{(0,0)}{\longrightarrow} 14 \stackrel{(1,1)}{\longrightarrow} 1 .
$$

The tile at position $\boldsymbol{n}$ in the tiling $x$ in Figure 2 is indeed $x_{\boldsymbol{n}}=1$.

Since $\Omega_{\mathcal{Z}}$ is minimal, we believe that Theorem 1 can be extended to all configurations in $\Omega_{\mathcal{Z}}$ provided an additional input is given. Moreover, we believe that Theorem 1 holds for a large family of self-similar subshifts and not only for Fibonacci-like examples, thus extending Cobham's theorem to $\mathbb{Z}^{2}$ and $\mathbb{Z}^{d}$. This asks for further research and is part of an ongoing work. 


\section{References}

[AA20] Shigeki Akiyama and Pierre Arnoux, editors. Substitution and tiling dynamics: introduction to self-inducing structures. CIRM Jean-Morlet Chair, Marseille, France, Fall 2017, volume 2273. Cham: Springer, 2020.

[AS03] Jean-Paul Allouche and Jeffrey Shallit. Automatic sequences. Cambridge University Press, Cambridge, 2003. Theory, applications, generalizations.

[BR10] Valérie Berthé and Michel Rigo, editors. Combinatorics, automata, and number theory, volume 135. Cambridge: Cambridge University Press, 2010.

[CKR10] Emilie Charlier, Tomi Kärki, and Michel Rigo. Multidimensional generalized automatic sequences and shape-symmetric morphic words. Discrete Math., 310(6-7):1238-1252, 2010.

[JR21] Emmanuel Jeandel and Michaël Rao. An aperiodic set of 11 Wang tiles. Advances in Combinatorics, January 2021.

[Lab19] Sébastien Labbé. A self-similar aperiodic set of 19 Wang tiles. Geom. Dedicata, 201:81-109, 2019.

[Lab20a] Sébastien Labbé. Optional SageMath Package slabbe (Version 0.6.2). https: //pypi.python.org/pypi/slabbe/, 2020.

[Lab20b] Sébastien Labbé. Three characterizations of a self-similar aperiodic 2dimensional subshift. December 2020. arXiv:2012.03892.

[Lab21] Sébastien Labbé. Substitutive Structure of Jeandel-Rao Aperiodic Tilings. Discrete Comput. Geom., 65(3):800-855, 2021.

[LR01] P. B. A. Lecomte and M. Rigo. Numeration systems on a regular language. Theory Comput. Syst., 34(1):27-44, 2001.

[RM02] Michel Rigo and Arnaud Maes. More on generalized automatic sequences. $J$. Autom. Lang. Comb., 7(3):351-376, 2002.

[Zec72] E. Zeckendorf. Représentation des nombres naturels par une somme de nombres de Fibonacci ou de nombres de Lucas. Bull. Soc. Roy. Sci. Liège, 41:179-182, 1972 . 


\section{Appendix}

We now define the notion of markers for subshifts $X \subset \mathcal{A}^{\mathbb{Z}^{d}}$. Their existence allows to desubstitute uniquely the configurations in $X$ using a $d$-dimensional morphism. Originally, those results were proved for $d=2$ in order to desubstitute configurations from Wang shifts, see [Lab19] and [Lab21]. It turns out that the notion of markers is more general and the results holds in general subshifts $X \subset \mathcal{A}^{\mathbb{Z}^{d}}[\mathrm{Lab} 20 \mathrm{~b}]$.

Recall that if $w: \mathbb{Z}^{d} \rightarrow \mathcal{A}$ is a configuration and $a \in \mathcal{A}$ is a letter, then $w^{-1}(a) \subset \mathbb{Z}^{d}$ is the set of positions where the letter $a$ appears in $w$.

Definition 15. Let $\mathcal{A}$ be an alphabet and $X \subset \mathcal{A}^{\mathbb{Z}^{d}}$ be a subshift. A nonempty subset $M \subset \mathcal{A}$ is called markers in the direction $\boldsymbol{e}_{i}$, with $i \in\{1, \ldots, d\}$, if positions of the letters of $M$ in any configuration are nonadjacent $(d-1)$-dimensional layers orthogonal to $\boldsymbol{e}_{i}$, that is, for all configurations $w \in X$ there exists $P \subset \mathbb{Z}$ such that the positions of the markers satisfy

$$
w^{-1}(M)=P \boldsymbol{e}_{i}+\sum_{k \neq i} \mathbb{Z} \boldsymbol{e}_{k} \quad \text { with } \quad 1 \notin P-P
$$

where $P-P=\{b-a \mid a \in P, b \in P\}$ is the set of differences between elements of $P$.

Note that it follows from the definition that a subset of markers is a proper subset of $\mathcal{A}$ as the case $M=\mathcal{A}$ is impossible.

The presence of markers allows to desubstitute uniquely the configurations of a subshift. There is even a choice to be made in the construction of the substitution. We may construct the substitution in such a way that the markers are on the left or on the right in the image of letters that are dominoes in the direction $\boldsymbol{e}_{k}$. We make this distinction in the statement of the following result which was stated in the context of Wang shifts in [Lab19,Lab21] and extended to $\mathbb{Z}^{d}$ in $[$ Lab20b].

Theorem 16. [Lab20b] Let $\mathcal{A}$ be an alphabet and $X \subset \mathcal{A}^{\mathbb{Z}^{d}}$ be a subshift. If there exists a subset $M \subset \mathcal{A}$ of markers in the direction $\boldsymbol{e}_{i} \in\left\{\boldsymbol{e}_{1}, \ldots, \boldsymbol{e}_{d}\right\}$, then

(i) (markers on the right) there exists an alphabet $\mathcal{B}_{R}$, a subshift $Y \subset \mathcal{B}_{R}^{\mathbb{Z}^{d}}$ and a 2-dimensional morphism $\omega_{R}: Y \rightarrow X$ such that

$$
\omega_{R}\left(\mathcal{B}_{R}\right) \subseteq(\mathcal{A} \backslash M) \cup\left((\mathcal{A} \backslash M) \odot^{i} M\right)
$$

which is recognizable and onto up to a shift and

(ii) (markers on the left) there exists an alphabet $\mathcal{B}_{L}$, a subshift $Y \subset \mathcal{B}_{L}^{\mathbb{Z}^{d}}$ and a 2-dimensional morphism $\omega_{L}: Y \rightarrow X$ such that

$$
\omega_{L}\left(\mathcal{B}_{L}\right) \subseteq(\mathcal{A} \backslash M) \cup\left(M \odot^{i}(\mathcal{A} \backslash M)\right)
$$

which is recognizable and onto up to a shift. 
The morphisms provided in Theorem 16 can be computed with an algorithm taking as input the subset of marker tiles. Those algorithms are available in [Lab21,Lab20b] and are implemented in the slabbe optional package [Lab20a] of SageMath. The algorithms are used in the proof below.

In the following proof, we use the notation $\llbracket m, n \rrbracket=\{k \in \mathbb{Z} \mid m \leq k \leq n\}$, where $m, n \in \mathbb{Z}$, to denote interval of integers.

Proof (of Proposition 10). We use the algorithms to desubstitute Wang shifts based on the notion of marker tiles [Lab21, Theorem 3.6]. We construct the set $\mathcal{Z}$ of 16 Wang tiles:

sage: from slabbe import WangTileSet

sage: tiles = ["DOJO", "DOHL", "JMDP", "DMDK", "HPJP", "HPHN", "HKDP", "BOIO",

....: "ILEO", "ILCL", "ALIO", "EPIP", "IPIK", "IKBM", "IKAK", "CNIP"]

sage: $\mathrm{Z}=$ WangTileSet ([tuple(tile) for tile in tiles]); $\mathrm{Z}$

Wang tile set of cardinality 16

We compute a subset of markers $M \subset \mathcal{Z}$ for the direction $\boldsymbol{e}_{2}$ and we use them to desubstitute the Wang shift $\Omega_{\mathcal{Z}}$ :

sage: $Z$.find_markers $(i=2$, radius $=2$, solver="dancing_links")

$[0,1,2,3,4,5,6]]$

sage: $M=[0,1,2,3,4,5,6]$

sage: $Z 1$, gamma $0=Z$.find_substitution $(M, i=2$, radius $=2$,

....: side="right", solver="dancing_links")

We obtain the morphism $\gamma_{0}: \Omega_{\mathcal{Z}_{1}} \rightarrow \Omega_{\mathcal{Z}}$ given as a rule of the form

$$
\begin{aligned}
& \gamma_{0}: \llbracket 0,17 \rrbracket \rightarrow \llbracket 0,15 \rrbracket^{*^{2}}
\end{aligned}
$$

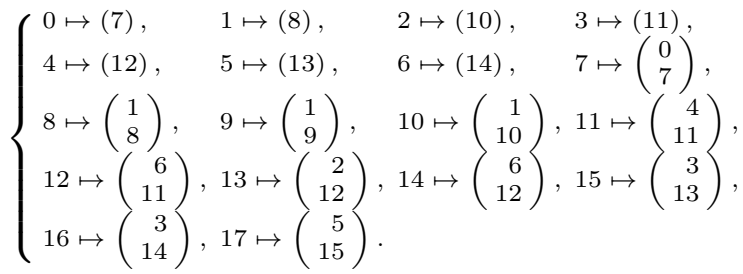

and the set $\mathcal{Z}_{1}$ of 18 Wang tiles

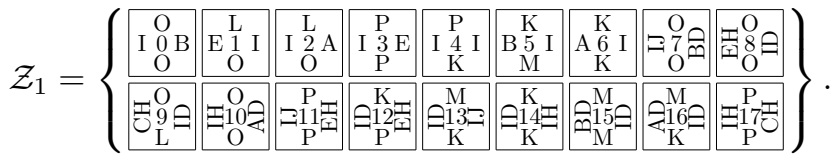

We compute a subset of markers $M_{1} \subset \mathcal{Z}_{1}$ for the direction $\boldsymbol{e}_{1}$ and we use them to desubstitute the Wang shift $\Omega_{\mathcal{Z}_{1}}$ :

sage: $Z 1$.find_markers ( $i=1$, radius=1, solver="dancing_links")

$[0,1,2,7,8,9,10]]$

sage: $M=[0,1,2,7,8,9,10]$

sage: $Z 2$,gamma1 $=Z 1$. find_substitution $(M, i=1$, radius $=1$

....: side="right", solver="dancing_links")

We obtain the morphism $\gamma_{1}: \Omega_{\mathcal{Z}_{2}} \rightarrow \Omega_{\mathcal{Z}_{1}}$ given as a rule of the form

$$
\begin{aligned}
& \gamma_{1}: \llbracket 0,15 \rrbracket \rightarrow \llbracket 0,17 \rrbracket^{*^{2}}
\end{aligned}
$$

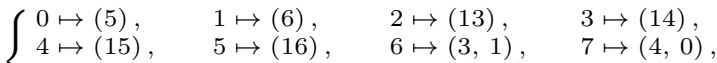

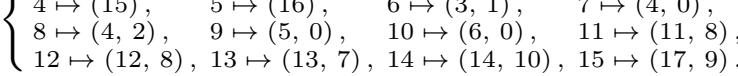


and the set $\mathcal{Z}_{2}$ of 16 Wang tiles

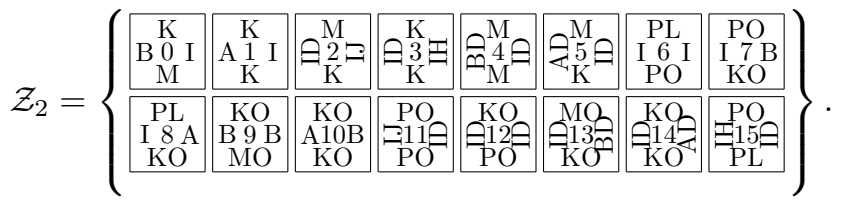

It turns out that $\mathcal{Z}$ and $\mathcal{Z}_{2}$ are equivalent. The proof can be done using graph isomorphisms:

sage: $\mathrm{Z2.is}$.equivalent( $\mathrm{Z}$ )

The bijection vert between the vertical colors, the bijection horiz between the horizontal colors and bijection $\gamma_{2}$ from $\mathcal{Z}_{2}$ to $\mathcal{Z}$ is computed as follows:

sage: _,vert,horiz, gamma2 $=$ Z.is_equivalent $(Z 2$, certificate=True)

We obtain the morphism $\gamma_{2}: \Omega_{\mathcal{Z}} \rightarrow \Omega_{\mathcal{Z}_{2}}$ given as a rule of the form

$$
\begin{aligned}
\gamma_{2}: \llbracket 0,15 \rrbracket \rightarrow \llbracket 0,15 \rrbracket^{*^{2}} \\
\left\{\begin{array}{llll}
0 \mapsto(1), & 1 \mapsto(0), & 2 \mapsto(8), & 3 \mapsto(6), \\
4 \mapsto(10), & 5 \mapsto(9), & 6 \mapsto(7), & 7 \mapsto(3), \\
8 \mapsto(5), & 9 \mapsto(4), & 10 \mapsto(2), & 11 \mapsto(14), \\
12 \mapsto(12), & 13 \mapsto(15), & 14 \mapsto(11), & 15 \mapsto(13) .
\end{array}\right.
\end{aligned}
$$

and we have

$$
\begin{aligned}
& \gamma_{0} \gamma_{1} \gamma_{2}: \llbracket 0,15 \rrbracket \rightarrow \llbracket 0,15 \rrbracket^{*^{2}}
\end{aligned}
$$

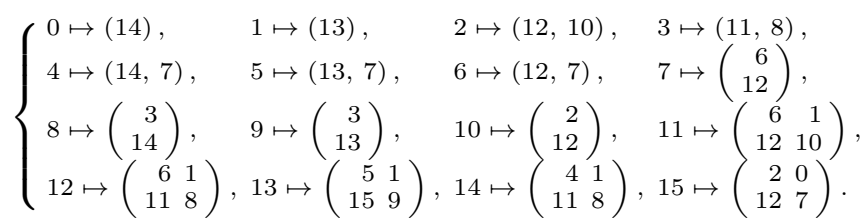

We conclude that $\Omega_{\mathcal{Z}}={\overline{\gamma_{0}\left(\Omega_{\mathcal{Z}_{1}}\right)}}^{\sigma}={\overline{\gamma_{0} \gamma_{1}\left(\Omega_{\mathcal{Z}_{2}}\right)}}^{\sigma}={\overline{\gamma_{0} \gamma_{1} \gamma_{2}\left(\Omega_{\mathcal{Z}}\right)}}^{\sigma}={\overline{\phi\left(\Omega_{\mathcal{Z}}\right)}}^{\sigma}$. 
Theorem 17. The Wang shift $\Omega_{\mathcal{Z}}$ generated by the tiles $\mathcal{Z}$ is self-similar, minimal and aperiodic.

Proof (of Theorem 17). From Proposition 10 we obtain that $\Omega_{\mathcal{Z}}$ is self-similar satisfying ${\overline{\phi\left(\Omega_{\mathcal{Z}}\right)}}^{\sigma}=\Omega_{\mathcal{Z}}$. Since $\phi$ is expansive and recognizable, the Wang shift $\Omega_{\mathcal{Z}}$ is aperiodic [Lab19, Proposition 6].

We compute the set of $2 \times 2$ words for which the morphism $\phi$ is prolongable for some power of $\phi$ in each of the four quadrants:

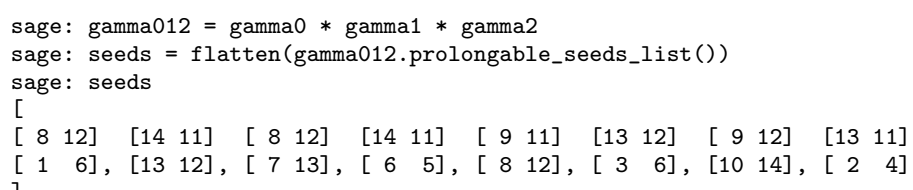

We check whether these $2 \times 2$ factors belong to the language $\mathcal{L}_{\phi}$ generated from the application of the morphism on the letters:

sage: seeds $=[[$ list (reversed $(\mathrm{col}))$ for col in seed.columns ()$]$ for seed in seeds]

sage: $F=$ gamma012. list_2x2_factors $($ )

sage: all (seed in $\mathrm{F}$ for seed in seeds)

True

Thus the substitutive subshift $\mathcal{X}_{\phi}$ is the unique self-similar subshift $X \subset \llbracket 0,15 \rrbracket^{\mathbb{Z}^{2}}$ satisfying $\overline{\phi(X)}^{\sigma}=X$ [Lab20b, Lemma 3.9]. Thus $\mathcal{X}_{\phi}=\Omega_{\mathcal{Z}}$. Moreover $\mathcal{X}_{\phi}$ is minimal since $\phi$ is primitive and expansive. 